\title{
Knuckle pads mimic early psoriatic arthritis
}

\author{
I. Giovannini ${ }^{1}$, S. Zandonella Callegher ${ }^{1}$, E. Errichetti ${ }^{2}$, S. De Vita ${ }^{1}$, A. Zabotti ${ }^{1}$ \\ ${ }^{1}$ Department of Medical and Biological Science, Rheumatology Clinic, University of Udine, Italy; \\ 2Institute of Dermatology, "Santa Maria della Misericordia" University Hospital, Udine, Italy
}

\section{SUMMARY}

Knuckle pads or Garrod's nodes are a rare, non-inflammatory condition. They consist of benign, well-circumscribed fibro-adipose tissue over the small joints of hands and feet. Knuckle pads may be under-diagnosed and mistaken for early arthritis. The rheumatologist should perform an accurate differential diagnosis in which he can be helped by ultrasound and by other colleagues, such as the dermatologist. Ultrasound is considered useful in the assessment of the thickening of the subcutaneous tissue, located usually on the extensor site of proximal interphalangeal and metacarpophalangeal hand joints. Dermoscopy may play a role in detecting epidermal and dermal changes. We hereby report the case of a female patient with knuckle pads mimicking psoriatic arthritis.

Key words: Knuckle pads, Garrod's nodes, psoriatic arthritis, ultrasound, dermoscopy.

Reumatismo, 2021; 73 (1): 67-69

\section{INTRODUCTION}

$\mathrm{T}$ he evaluation of small joint swelling in young patients is often challenging. This clinical manifestation should be differentiated from a joint disease as well as from lesions of the para-articular tissues, such as soft tissue growth or other masses. In young patients, the swelling of the small joints of the hands is usually related to inflammatory diseases (e.g., psoriatic arthritis, rheumatoid arthritis, connective tissue diseases), but also non-inflammatory conditions should be considered in the differential diagnosis. Non-inflammatory diseases presenting with swelling of the small joints of the hands could be caused by a local lesion, such as synovial cyst and giant cell tumor or a systemic disease (e.g., acromegaly).

Imaging, such as magnetic resonance or ultrasonography (US), is useful in differential diagnosis, particularly in doubtful cases. US, which is nowadays part of routine clinical practice especially in early arthritis management, could assist the clinician to identify the involved structures and the degree of inflammation and damage, even in the pre-clinical phases of psoriatic arthritis (1).

\section{CASE REPORT}

We report the case of a 30-year-old previously healthy woman with reddish, occasionally painful swelling of the right hand proximal interphalangeal (PIP) joints, who presented to our rheumatology clinic to have a second opinion for a suspected psoriatic arthritis (Figure 1a).

The swelling appeared a year earlier, initially in the $2^{\text {nd }}$ and $4^{\text {th }}$ right PIP joints, then extended to the $3^{\text {rd }}$ and $5^{\text {th }}$ right hand PIP joints, associated with redness and local pain (Figure 1a). The patient's sister suffered from psoriasis and their mother had frequently uveitis. Blood investigations revealed negative anti-nuclear antibodies, extractable nuclear antigen panel, rheumatoid factor, anti-citrullinated peptide antibodies and C-reactive protein. The patient complained about local mild pain symptoms and had no signs of inflammation in PIP joints. No other joint was affected. Because of the swelling with redness and the family history of psoriasis, psoriatic arthritis was suspected, and sulfasalazine $2 \mathrm{gr} / \mathrm{die}$ was proposed. However, the patient reported no improvement.

US and dermoscopy were used for the differential diagnosis of joint swelling. US
Corresponding author: Salvatore De Vita Rheumatology Clinic,

Santa Maria della Misericordia,

University Hospital, Italy

E-mail: salvatore.devita@asufc.sanita.fvg.it 
(Samsung RS 85) highlighted a hypoechoic area over the central slip of the third right extensor tendon, without joint involvement and without any signs of synovitis or enthesitis (Figure 1b). Dermoscopy revealed lesions with white scaling and white-yellow areas, histologically corresponding to hyperkeratosis and underlying fibrosis, respectively (Figure 1c). Subsequently, the diagnosis of psoriatic arthritis was not confirmed, based on clinical signs and imaging features, whereas knuckle pads were diagnosed. The patient was successfully treated with a combination of topical cantharidin -odophyllotoxin - salicylic acid.
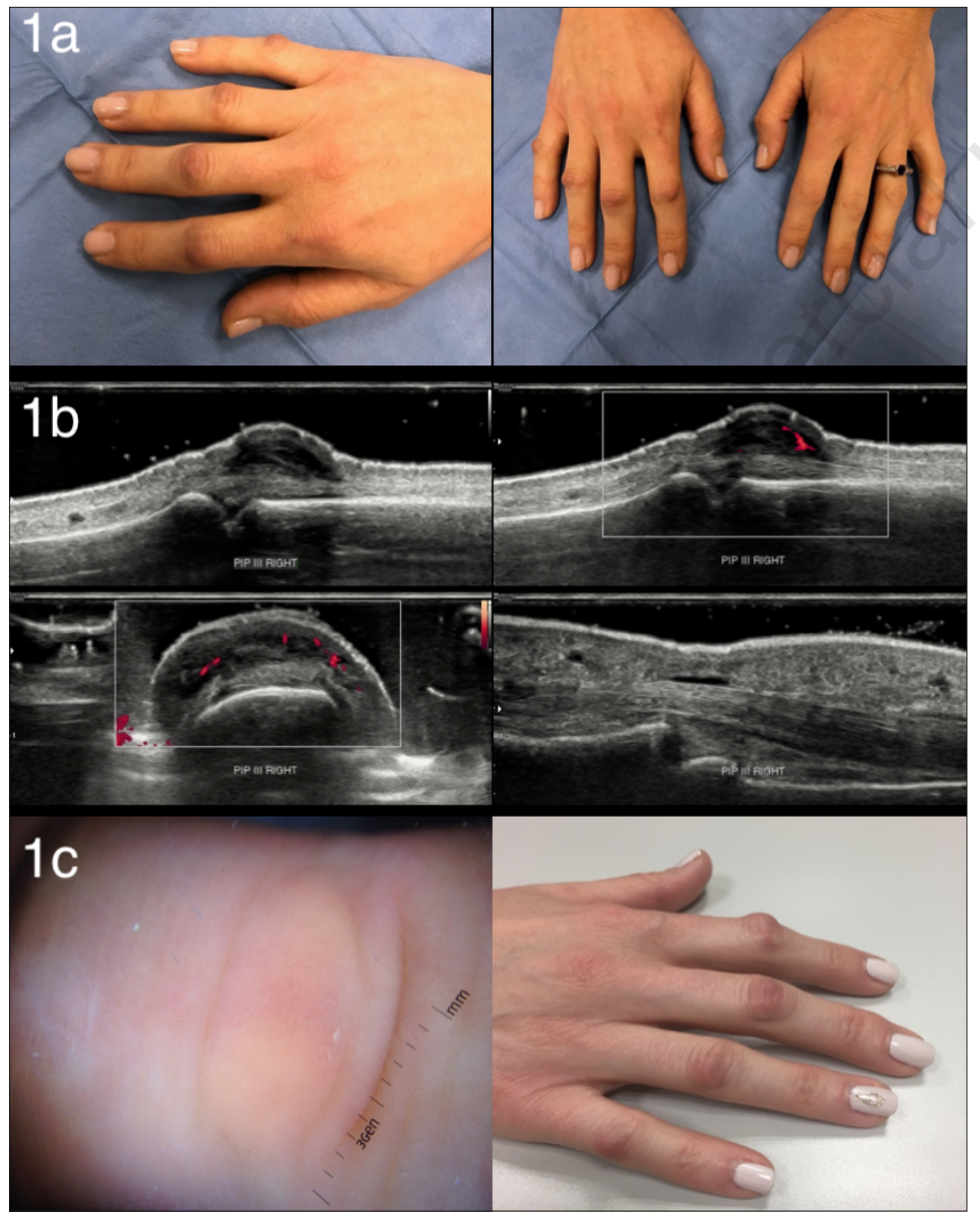

Figure 1 - a) Knuckle pad lesions over the finger joints (proximal interphalangeal joints of the right hand); b) Ultrasonographic assessment of knuckle pads: hypoechoic area over the central slip of the third right extensor tendon. Joint and tendon involvement were not detected; c) Dermoscopic evaluation of knuckle pads revealing epidermal and dermal changes (left). Knuckle pads dermatologic clinical evaluation (right).

\section{DISCUSSION}

Knuckle pads are benign subcutaneous nodules that appear most frequently on the small joints of the hands. In children, they are often idiopathic, and no universally effective treatment has been reported. Knuckle pads or Garrod's nodes are benign fibro-adipose pads located in the subcutaneous tissues over the small joints of hands and feet and could be mistaken for arthritis or dermal lesions $(2,3)$.

These lesions usually present as slowly enlarging, painless pads, typically located on the extensor site of the small joints of the hands [in particular PIP and metacarpophalangeal (MCP) joints]. Knuckle pads have been associated with repetitive friction or pressure (e.g., habitual chewing or sucking of the fingers, repetitive occupational activities, athletic activities like boxing and surfing and bulimia nervosa with self-induced vomiting), fibrosing disorders (Dupuytren's contracture, Ledderhose's syndrome/plantar fascial fibromatosis and Peyronie's disease) and autosomal dominant syndrome (Bart-Pumphrey syndrome), but idiopathic cases have also been described (4).

In our patient ultrasonography showed a hypoechoic subcutaneous thickening on the dorsal side of affected MCP or PIP joints. These pads had a dome shape with irregular borders. Generally, they consist of non-compressible nodules without power Doppler signal, unlike what happens in psoriatic arthritis (4). Some hypervascularization may be rarely detected in the peripheral area (5) (Figure 1). The adjacent joints and tendons are usually normal.

As recommended for PsA, a clinical and ultrasound examination of the affected region with a high-resolution ultrasonography is needed to perform an accurate assessment of the joints and adjacent soft tissues (6). Also a joint rheumatological and dermatological assessment can be useful (7). Dermoscopy may also be helpful $(8,9)$, as it may show epidermal alterations (scaling due to hyperkeratosis) and dermal alterations (white-yellow areas corresponding to fibrosis), which reflect the his- 
tological alterations seen in knuckle pads (10). There is no effective treatment for knuckle pads (11). Behavior changes and psychiatric support may show results, otherwise watchful waiting is recommended. Some case report described some efficacy of local treatments, such as intralesional fluorouracil or antharidin-podophylotoxinsalicylic acid $(12,13)$.

\section{CONCLUSIONS}

In conclusion, in cases of joint swelling of the hand joints mimicking arthritis, careful history taking may suggest non-inflammatory diseases. The diagnosis of knuckle pads can be readily made by clinical examination and ultrasonography. Dermoscopy might also be useful to assist clinicians in doubtful cases.

\section{Conflict of interests}

The authors declare no potential conflict of interests.

\section{REFERENCES}

1. Zabotti A, McGonagle DG, Giovannini I, et al. Transition phase towards psoriatic arthritis: clinical and ultrasonographic characterisation of psoriatic arthralgia. RMD Open. 2019; 5: e001067.

2. Nenoff P, Woitek G. Knuckle Pads [Internet]. Massachusetts Medical Society; 2011 [cited 2021 Jan 6]. Available from: https://www. nejm.org/doi/10.1056/NEJMicm1008915.

3. Zabotti A, Errichetti E, Cereser L, et al. Pachy- dermodactyly: the role of ultrasonography and dermoscopy for diagnosis. Rheumatology. 2017; 56: 703-703.

4. Hyman $\mathrm{CH}$, Cohen PR. Report of a family with idiopathic knuckle pads and review of idiopathic and disease-associated knuckle pads. Dermatol Online J 2013; 19: 18177.

5. Lopez-Ben R, Dehghanpisheh K, Chatham WW, et al. Ultrasound appearance of knuckle pads. Skeletal Radiol. 2006; 35: 823-7.

6. Zabotti A, Piga M, Canzoni M, et al. Ultrasonography in psoriatic arthritis: which sites should we scan? Annals of the Rheumatic Diseases. 2018; 77: 1537-8.

7. Savage L, Tinazzi I, Zabotti A, et al. Defining pre-clinical psoriatic arthritis in an integrated dermato-rheumatology environment. J Clin Med Multidis Digital Publ Inst. 2020; 9: 3262.

8. Zabotti A, Errichetti E, Zuliani F, et al. Early psoriatic arthritis versus early seronegative rheumatoid arthritis: role of dermoscopy combined with ultrasonography for differential diagnosis. J Rheumatol. 2018; 45: 648-54.

9. Errichetti E, Zabotti A, Stinco G, et al. Dermoscopy of nail fold and elbow in the differential diagnosis of early psoriatic arthritis sine psoriasis and early rheumatoid arthritis. J Dermatol. 2016; 43: 1217-20.

10. Kodama BF, Gentry RH, Fitzpatrick JE. Papules and plaques over the joint spaces. Knuckle pads (heloderma). Arch Dermatol. 1993; 129: 1044-5, 1047

11. Keersmaeker AD, Vanhoenacker F. Imaging Features of Knuckle Pads. J Belgian Soc Radiol. 2016; 100: 67.

12. Hasbún C, Sandoval M, Curi M. A novel treatment for idiopathic knuckle pads with cantharidin-podophylotoxin-salicylic acid. Pediatr Dermatol. 2019; 36: 544-5.

13. Weiss E. A novel treatment for knuckle pads with intralesional fluorouracil. Arch Dermatol. 2007; 143: 1447. 